\title{
POSNER'S SECOND THEOREM DEDUCED FROM THE FIRST
}

\author{
MARTIN MATHIEU
}

(Communicated by Maurice Auslander)

\begin{abstract}
Posner's second theorem is derived as a consequence of his first theorem.
\end{abstract}

In a paper from 1957, Posner proved the following two theorems [5]. We say that a mapping $f$ on a ring $R$ is centralizing if $[a, f(a)]=a f(a)-f(a) a \in$ $Z(R)$, the center of $R$, for all $a \in R$.

Theorem 1. Let $R$ be a prime ring with characteristic different from 2 . The product of two nonzero derivations of $R$ is not a derivation.

Theorem 2. Let $R$ be a prime ring. If there is a nonzero centralizing derivation of $R$, then $R$ is commutative.

These results were subsequently refined and extended by a number of authors; we refer to [4] for a state-of-the-art account and a comprehensive bibliography. While the proof of Theorem 1 consists in a straightforward computation, the second theorem has been considered to lie somewhat deeper although the arguments were simplified in [2]. In the present note we show how to obtain Theorem 2 directly from Theorem 1 , at least where it can be done, viz. if char $R \neq 2$. The exceptional case of characteristic 2 is, however, easily treated (see $[1$, p. 13]). In fact, since the arguments in the proof of Theorem 1 go over verbatim to the case where the hypothesis is only made on a nonzero ideal $I$ of $R$, we also obtain Theorem 2 under the assumption that the derivation is centralizing on $I$ only (cf. [4, Corollary, p. 283]).

Proof. Let $I$ be a nonzero ideal of the prime ring $R$ with char $R \neq 2$, and let $\delta: R \rightarrow R$ be a derivation such that $[a, \delta a] \in Z(R)$ for all $a \in I$. Replacing $a$ by $a+b, b \in I$, yields

$$
[a, \delta b]+[b, \delta a] \in Z(R),
$$

in particular,

$$
\left[a, \delta\left(a^{2}\right)\right]+\left[a^{2}, \delta a\right] \in Z(R) .
$$

Received by the editors July 17, 1990 and, in revised form, October 26, 1990.

1980 Mathematics Subject Classification (1985 Revision). Primary 16A12; Secondary 16A70, 16A72.

Key words and phrases. Prime ring, centralizing derivation.

This paper was written during a visit to Dalhousie University, Halifax, Canada. 
As $\delta$ is a derivation, it is easily computed that

$$
\left[a, \delta\left(a^{2}\right)\right]-\left[a^{2}, \delta a\right]=0,
$$

and therefore combining (2) and (3) gives $\left[a^{2}, \delta a\right] \in Z(R)$. Since $[a, \delta a]$ is central too, we thus obtain

$$
2[a, \delta a]^{2}=[2[a, \delta a] a, \delta a]=\left[\left[a^{2}, \delta a\right], \delta a\right]=0,
$$

and therefore $[a, \delta a]=0$ as $R$ is prime.

This shows that in (1) we in fact have

$$
[a, \delta b]+[b, \delta a]=0 \text { for all } a, b \in I .
$$

Let $\delta_{c}$ denote the inner derivation $\delta_{c}(x)=[x, c], x \in R$. Then $\left(1^{\prime}\right)$ says nothing but $\delta_{b} \circ \delta=\delta_{\delta b}$ on $I$ for all $b \in I$. By Theorem 1, we conclude that $\delta=0$ or $\delta_{b}=0$ for all $b \in I$, i.e. $I \subseteq Z(R)$. Since $R$ is prime, the latter case entails that $R$ is commutative.

Remarks. 1. Centralizing derivations are commuting (that is $[a, \delta a]=0$ for all $a$ ) under rather general assumptions [3, Lemma 4]; this was kindly pointed out to us by the referee.

2. If char $R=2$, then (1) implies ( $1^{\prime}$ ) even more easily, cf. [1]. Bergen's version of Theorem 1 in the characteristic 2 case [4, Theorem 4] thus yields an element $c$ in the extended centroid of $R$, such that $\delta_{b}=c \delta$ for all $b \in I$, which is only possible if $\delta_{b}=0$ for all $b$. Therefore, the exceptional case can be treated in the same manner.

\section{REFERENCES}

1. M. Ahmad, On a theorem of Posner, Proc. Amer. Math. Soc. 66 (1977), 13-16.

2. R. Awtar, On a theorem of Posner, Proc. Cambridge Phil. Soc. 73 (1973), 25-27.

3. H. E. Bell and W. S. Martindale, Centralizing mappings of semiprime rings, Canad. Math. Bull. 30 (1987), 92-101.

4. C. Lanski, Differential identities, Lie ideals, and Posner's theorems, Pacific J. Math. 134 (1988), 275-297.

5. E. C. Posner, Derivations in prime rings, Proc. Amer. Math. Soc. 8 (1957), 1093-1100.

Mathematisches Institut der Universität TÜbingen, AUf Der Morgenstelle 10, D-7400 TÜBINGen, Federal RePUblic OF Germany 